\title{
NUEVOS ESTUDIOS DEL ESQUELETO DEL SITIO CERRO YANQUENAO (COLHUE HUAPI, CHUBUT). VEINTIOCHO AÑOS DESPUÉS
}

\author{
SOLANA GARCIA GURAIEB*, VALERIA BERNAL", \\ PAULA. N. GONZÁLEZ"*, LUIS A. BOSIO"** Y ANA M. AGUERRE"***
}

\begin{abstract}
RESUMEN
El objetivo de esta comunicación es presentar nuevos datos bioarqueológicos sobre los restos óseos humanos procedentes del sitio arqueológico Cerro Yanquenao. El entierro corresponde a un individuo de sexo masculino de entre 40 y 50 años de edad, que presenta deformación de tipo tabular erecto, variedad plano-frontal. La datación obtenida indicó una antigüedad $1.151 \pm 59$ años radiocarbónicos AP. Los valores de $\delta C^{13}(-18,5 \%)$ y de $\delta \mathrm{N}^{15}(11,5 \%)$ se encuentran dentro de los rangos esperados para dietas continentales terrestres en Patagonia. Se identificaron distintas lesiones patológicas. Las más conspicuas corresponden a lesiones óseas en los miembros inferiores y superiores y en el cráneo que podrían ser compatibles con alguna variedad de treponematosis. Los resultados generales obtenidos se discuten en relación con los problemas de investigación y la información bioarqueológica actualmente vigente en la región.
\end{abstract}

PALABRAS CLAVE: Bioarqueología, cazadores-recolectores, Patagonia.

\section{"NEW STUDIES ON THE SKELETON FROM CERRO YANQUENAO (COLHUE HUAPI, CHUBUT). TWENTY-EIGHT YEARS AFTER”}

\begin{abstract}
The purpose of this communication is to present a reassessment of bioarchaeological data of the skeletal remains from the archaeological site Cerro Yanquenao (Chubut, Argentina). The new studies indicate that this individual is a male, aged between 40-50 years at the time of death. The cranium shows artificial deformation of tabular erect type, planolambdic variety. A fragment of rib analyzed by AMS indicated an age of $1.151 \pm 59$ years BP. $\delta C^{13}(-18.5 \%)$ and $\delta \mathrm{N}^{15}(11.5 \%)$ values are within the range expected for terrestrial diets in continental Patagonia. Also, several bony lesions were identified. The

* CONICET- INAPL. 3 de febrero 1378 (C1426BJN). C. A. Bs. As. solanagg@gmail.com. Argentina.

* CONICET- UNLP. Facultad de Ciencias Naturales y Museo. Universidad Nacional de La Plata. bernalv@fcnym.unlp.edu.ar, pgonzalez@fcnym.unlp.edu.ar. Argentina.

Cuerpo Médico Forense/UBA. Junin 760. (C1026ABP) C.A.Bs.As. luisbosio@yahoo.com.ar. Argentina.

**** CONICET-UBA- Instituto de Arqueología, Facultad de Filosofía y Letras, Universidad de Buenos Aires. amaguerre@gmail. com. Argentina.
\end{abstract}


most conspicuous ones were found in the upper and lower limbs and the skull and might be compatible with a variety of Treponematosis. These results are discussed in relation to the current approaches and bioarchaeological information known for the region.

KEY WORDS: Bioarchaeology, hunter-gatherers, Patagonia.

\section{INTRODUCCIÓN}

El objetivo de esta comunicación es presentar nuevos datos bioarqueológicos sobre los restos óseos humanos procedentes del sitio arqueológico Cerro Yanquenao, Provincia de Chubut. El enterratorio o "chenque" fue hallado por Carlos J. Gradin en 1979, quien solicitó a la Prof. Josefa. Patti de Martinez Soler y a la Lic. Carmen Vayá, los estudios bioantropológicos que fueron integrados a la publicación sobre el sitio, editado por el Gobierno de la Provincia (Gradin 1986).

Uno de nosotros, Ana M. Aguerre, se interesó desde el 2003, en retomar los estudios en el sur del Chubut. En este marco, uno de los objetivos fue emprender la realización de nuevos análisis del esqueleto de Cerro Yanquenao, que permitieran, a través de la aplicación de una metodología diferente y nuevas técnicas, una reevaluación del mismo a la luz de lo conocido en la actualidad sobre el contexto bioarqueológico regional. De este modo, casi treinta años después del primer estudio, se presentan aquí estos análisis, que incluyen el fechado radiocarbónico directo del esqueleto, análisis de isótopos estables para la estimación del tipo de dieta y una reevaluación del perfil biológico del individuo. Esto nos permitirá actualizar los estudios realizados previamente en relación con los problemas de investigación y la información bioarqueológica actualmente conocidas en la región.

\section{EL SITIO}

El cerro Yanquenao, ubicado aproximadamente a los 450 06' Lat. Sur y 6851' Long. Oeste, se halla a $85 \mathrm{~km}$ de la localidad de Colonia Sarmiento, al norte del Lago Colhue Huapi en la Provincia del Chubut (Gradin 1986). El sitio fue localizado en el año 1970 por C. J. Gradin y equipo. Al pie del faldeo se encontró material lítico y fragmentos de cerámica. El hallazgo más notable fueron numerosos bloques rocosos con arte rupestre del "estilo de grabados", distribuidos entre la base y la cima (Gradin 1986: 11 a 36). Posteriormente, durante la campaña en 1979, el equipo halló este entierro, a unos trescientos metros al noreste del cerro Yanquenao, en una barda basáltica cuya posición altimétrica era aproximadamente la misma que la cumbre del cerro, a 600 msnm. El entierro corresponde a los denominados de tipo chenque. La acumulación de rocas que conformaba la estructura presentaba forma oval de aproximadamente $1,90 \mathrm{~m} \times 1,20 \mathrm{~m}$ y su eje mayor mostraba una orientación NO-SE. La excavación del chenque permitió recuperar el entierro primario de un individuo adulto cuyo esqueleto mostraba un alto grado de articulación y de integridad. Se hallaba depositado superficialmente, en posición decúbito lateral izquierdo, con los miembros inferiores flexionados en un ángulo de aproximadamente $90^{\circ}$ y los miembros superiores flexionados sobre el pecho. No se encontró ningún tipo de acompañamiento junto al individuo -e.g. ajuar funerario u otro material arqueológico asociado- (Gradin 1986: pp.9-12).

\section{ESTUDIOS PREVIOS DEL ESQUELETO HUMANO DEL CERRO YANQUENAO}

Los estudios realizados por la Prof. Josefa Patti de Martínez Soler y la Lic. Carmen Vayá (1986) consistieron en la estimación del sexo, la edad y la estatura del individuo y en un detallado relevamiento métrico craneal y postcraneal. Las determinaciones indicaron que el esqueleto se encontraba en buen estado de conservación y mostraba un alto grado de completitud. El sexo del individuo fue determinado como masculino de acuerdo con la morfología del cráneo, el atlas y los coxales. Las estimaciones de edad realizadas sobre la base del grado de cierre de las suturas de la bóveda craneana y del desgaste dental indicaron que el mismo habría tenido entre 25-30 años de edad aproximadamente. También identificaron la presencia de deformación intencional del cráneo y la clasificaron como de tipo tabular erecto. A partir de los estudios métricos, 
las autoras señalaron también la alteración de los diámetros longitudinales y transversales de un lateral del esqueleto en relación con el otro, atribuyéndolo a la posible presencia de caracteres patológicos en el postcráneo. Por último, estimaron la estatura del individuo sobre la base de la longitud de los huesos largos (exceptuando el húmero que presenta una importante diferencia de longitud entre el lado derecho y el lado izquierdo) y el empleo de la tabla de Manouvrier. Estos resultados dieron como promedio una estatura de 1,66 m. Como apéndice a su trabajo, incluyeron el trabajo de Silvia Miranda, Odontóloga (1986: 61 a 62) quien realizó el análisis dental del esqueleto. Este estudio determinó la pérdida de varias piezas dentales postmortem y otras antemortem, estas últimas como producto de la enfermedad periodontal; asimismo, se registró la presencia de tártaro (i.e. sarro) en varias de las piezas dentales restantes.

\section{NUEVOS ESTUDIOS: VEINTIOCHO AÑOS DESPUÉS}

En esta oportunidad se realizaron estudios bioarqueológicos que incluyeron la realización de un inventario de los elementos óseos presentes, la determinación del sexo probable, la estimación de la edad al momento de la muerte, el relevamiento de deformación craneana artificial, la obtención de medidas antropométricas postcraneales, la estimación de la estatura y la evaluación de las patologías óseas y dentales presentes. Asimismo se obtuvo un fechado por AMS y se analizaron los isótopos estables del carbono y el nitrógeno $\left(\delta \mathrm{C}^{13}\right.$ y $\left.\delta \mathrm{N}^{15}\right)$. Cabe señalar que los estudios previos de 1980 no fueron consultados, hasta terminada la reevaluación del esqueleto realizada durante el 2007, para asegurar el mayor grado de independencia posible entre los resultados de ambos estudios. La metodología empleada en cada caso se describe a continuación.

Para la determinación del sexo probable se consideraron los criterios propuestos por Phenice (1969) y por Buikstra y Ubelaker (1994) para el análisis de los coxales, y los criterios de Acsádi y Nemeskéri (1970) para el cráneo. También se obtuvieron datos métricos tales como el diámetro máximo de la cabeza del fémur y del húmero (Ubelaker 1989) a partir de los cuales se estimó el sexo mediante el empleo de funciones discriminantes desarrolladas para muestras osteológicas de la región (Béguelin 2009; Béguelin y González 2008). La estimación de la edad probable de muerte se realizó a través de los indicadores de cambios morfológicos de la superficie auricular del ilion desarrollados por Lovejoy et al. (1985) y Meindl y Lovejoy (1989) y el grado de cierre de las suturas craneanas (Meindl y Lovejoy 1985). Debido a la fragmentación y/o ausencia de la sínfisis púbica en ambos coxales, no fue posible emplear como criterio ninguno de los métodos diseñados para los cambios morfológicos de la sínfisis púbica. La estatura se estimó a través de las formulas de regresión generadas por Béguelin (2005) para el fémur, ya que las mismas han sido diseñadas a partir de colecciones de esqueletos arqueológicos de Santa Cruz y Chubut. La evaluación y clasificación de la deformación craneana se realizó siguiendo los criterios de Imbelloni (1924-1925).

El estudio de las alteraciones patológicas se efectuó de forma macroscópica. Se tuvieron en cuenta en primer lugar, diversos indicadores inespecíficos de estrés habitualmente relevados en los estudios paleopatológicos tales como hipoplasias de esmalte dental, hiperostosis porótica y criba orbitaria, reacciones subperiósticas, artropatías y traumas ${ }^{1}$. Además, se efectuó un completo relevamiento de lesiones óseas siguiendo los lineamientos de Lovell (2000), que permitiera detectar otro tipo de patologías o contribuir al refinamiento de la clasificación etiológica de las lesiones observadas. Por último, se relevaron diversos indicadores de salud bucal (caries, abscesos, pérdida de dientes antemortem y enfermedad periodontal) empleando la metodología propuesta por Hillson (2000) y Clarke y Hirsch (1991). El desgaste dental se relevó de acuerdo con la escala de Smith (1984) para los molares y la de Scott (1979) para los dientes restantes.

La determinación de la edad radiocarbónica (AMS) fue realizada en el Arizona Radiocarbon AMS Facility (University of Arizona) a partir del colágeno de un fragmento óseo de costilla. La estimación paleodietaria fue realizada a partir del el análisis de $\delta \mathrm{N}^{15}$ y de $\delta \mathrm{C}^{13}$ en el mismo laboratorio y empleando la misma muestra.

1 La metodología general del estudio de estos indicadores paleopatológicos (definición, método de registro y cuantificación) ha sido desarrollada en otros trabajos (García Guraieb 2004, 2006) a los que se remite al lector por cuestiones de espacio. 


\section{RESULTADOS Y DISCUSIÓN}

\section{Fechado radiocarbónico}

El fechado radiocarbónico por AMS obtenido sobre un fragmento de costilla indicó una antigüedad de $1.151 \pm 59$ años AP (AA71849; 921-1171 calib². AP). A este respecto, cabe destacar que la modalidad de inhumación de tipo chenque, ampliamente difundida en Patagonia, parecería comenzar a registrarse, especialmente en el sur de Chubut y norte de Santa Cruz, a partir del último milenio -o unos pocos siglos antes-, de acuerdo con los fechados de los que se dispone actualmente en la región (i.e. entre ca. 1200 y 350 años radiocarbónicos AP; Goñi y Barrientos 2004). En este sentido, el chenque de Cerro Yanquenao, parecería corresponder a los momentos más tempranos de ocurrencia de esta modalidad funeraria y sería homologable al caso del chenque más antiguo, el chenque SAC 1-7, registrado en la cuenca del Salitroso, en el noroeste de Santa Cruz (Goñi y Barrientos 2004).

\section{Análisis isotópicos}

En cuanto a los análisis isótopicos realizados sobre la misma muestra (AA71849), se obtuvieron valores de de $\delta \mathrm{C}^{13}$ de $-18,5 \%$ o de $\delta \mathrm{N}^{15}$ de $11,5 \%$. Estos valores, se encuentran dentro de los rangos esperados para dietas continentales terrestres en Patagonia (Tessone et al. 2005) y son también homologables a los valores obtenidos para otros individuos en la región tanto para cronologías tardías, como la de Cerro Yanquenao, como para cronologías un poco más tempranas como la del sitio El Rodeo (esqueleto $\mathrm{N}^{\circ} 2$ ), a los $46^{\circ} 53^{\prime}$ de Lat. Sur y $70^{\circ} 0$ 'de Long. Oeste, en el área del río Pinturas, con un fechado de 1380 90 años AP (1054-1399 calib. AP) y un valor promedio de $\delta \mathrm{C} 13 \mathrm{de}-18.5$ \%o (Gradin y Aguerre 1994: 268 y Fernández y Panarello 1994: 304).

2 La calibración del fechado radiocarbónico del individuo de Cerro Yanquenao y del resto de las fechas citadas en este trabajo se realizó con el programa CALIB. REV 5.0.1. (1986-2005; basado en Stuiver M. y P.J. Reimer 1993). La calibración se realizó en años AP, con dos desvíos estándar, empleando la curva de calibración para el Hemisferio Sur (SHCal04) de Mc. Cormac et al. 2004.
Inventario y estado de conservación del esqueleto

El esqueleto está prácticamente completo. En la Tabla 1 se presenta el inventario y grado de conservación de las piezas óseas presentes en el individuo.

Determinación del sexo y estimación de la edad

De acuerdo con los criterios establecidos se trataría de un individuo de sexo masculino de entre 40 y 50 años de edad al momento de la muerte. El presente resultado difiere considerablemente del obtenido previamente por Patti de Martínez Soler y Vayá (1986: 41 y 42; edad estimada de 25 años) a partir de la observación de las suturas del cráneo. Esta diferencia puede atribuirse a que el método empleado previamente presenta rangos de variabilidad amplios, y como sugieren sus creadores, resulta más confiable cuando es utilizado en combinación con otros indicadores (Meindl y Lovejoy 1985). Por lo tanto el mayor número de rasgos empleados en este trabajo ha permitido obtener una estimación más precisa de la edad de muerte del individuo.

\section{Deformación craneana}

Siguiendo la clasificación Imbelloni (19241925), el individuo de Cerro Yanquenao presentaría deformación de tipo tabular erecto, variedad planofrontal (lo cual resulta coincidente con la determinación obtenida previamente por Patti de Martínez Soler y Vayá 1986:51 y 58). La variedad plano-frontal es común en Pampa y Patagonia; es la que presentan la mayoría de los cráneos deformados del Lago Buenos Aires, Provincia de Santa Cruz (Imbelloni 1923) y algunos de los cráneos deformados recuperados en la zona de Colhué Huapí, cercana al hallazgo de este individuo (Bórmida 1953- 1954). No obstante, en este último caso, coexisten también cráneos no deformados y cráneos con variedad plano-lámbdica. Lamentablemente, ninguno de estos esqueletos cuenta con fechados radioacarbónicos, pero el hecho de que todos ellos hallan sido recuperados en entierros de tipo chenque, permite una aproximación cronológica relativa que los ubicaría en el lapso correspondiente aproximadamente al segundo milenio AP. Esta observación es confirmada por los fechados radiocarbónicos obtenidos para esta 
Tabla 1. Inventario de elementos presentes del esqueleto de Cerro Yanquenao.

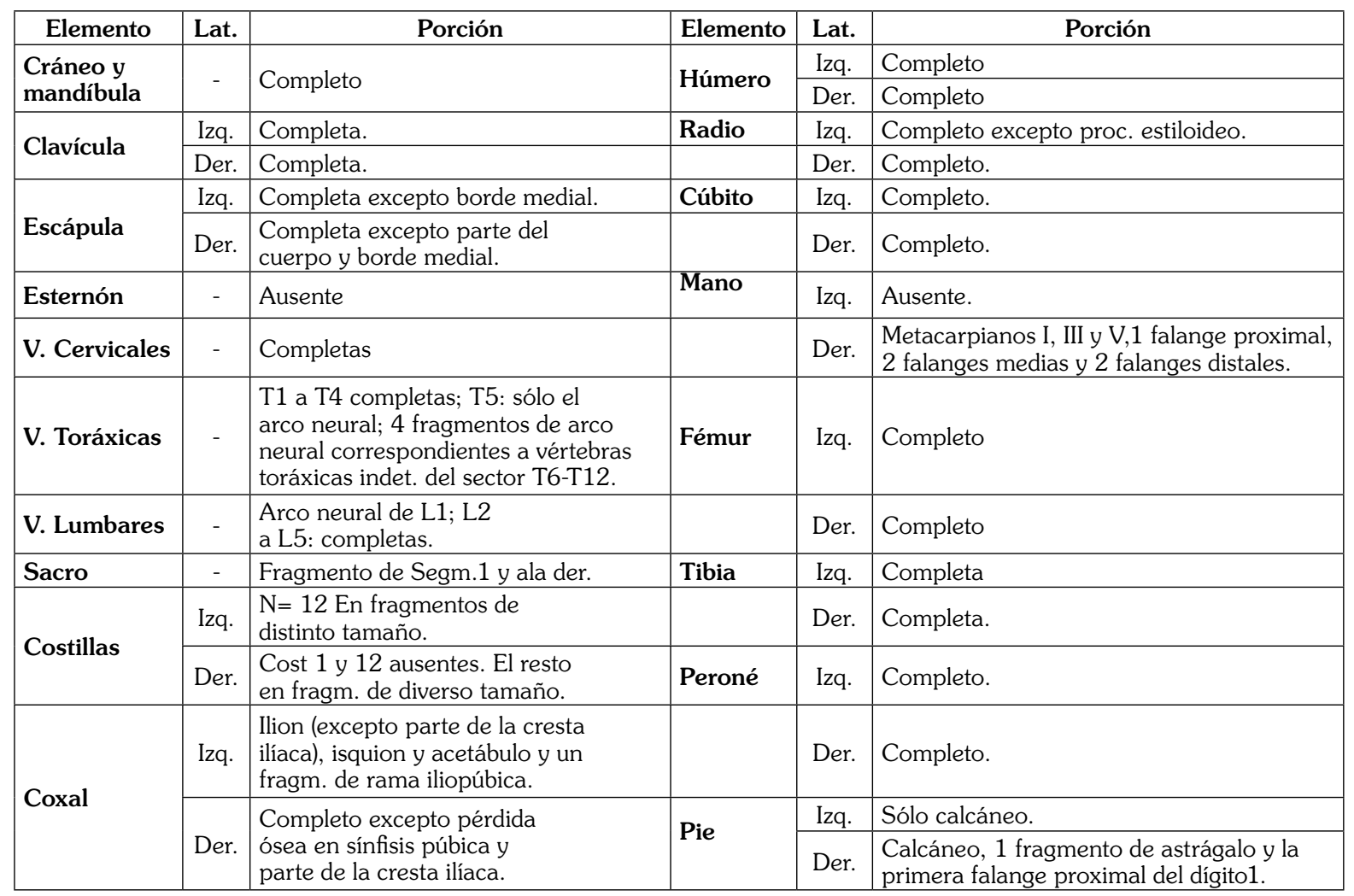

variedad deformatoria en los sitios: Alero Manantiales, ubicado en Río Mayo en el SO de Chubut (1.174 \pm 43 años AP; 955-1144 calib. AP, Bernal y Aguerre 2008), Sierra Colorada 1-7 (SAC 1-7), en la cuenca del Lago Salitroso en el NO de Santa Cruz (1.142 \pm 42 años AP; 923 -1082 calib. AP; Goñi et al. 2000-2002), en la desembocadura del río Chubut en el sitio "El Elsa" (1.990 \pm 60 años AP; 1714-2004 calib. AP) y en la Costa del Golfo Nuevo en el sitio "Playa del Pozo" (1.540 \pm 50 años AP; $1297-1424$ calib. AP) (Gómez Otero y Dahinten 1997-1998). La distribución de esta deformación es acotada a esta región no encontrándose en el resto del sector sur de Sudamérica (Perez 2007). En suma, los fechados radiocarbónicos obtenidos en este trabajo coinciden con los informados por estos autores, ubicando esta deformación entre 1000 y 2000 años AP.

\section{Antropometría y determinación de estatura}

Dada la exhaustividad del trabajo antropométrico realizado por las investigadoras mencionadas, sólo se presentarán aquí los nuevos datos antropométricos tomados para la longitud máxima de los huesos largos (Tabla 2) y los diámetros máximos de la cabeza del fémur y húmero.

Tabla 2. Longitud máxima de los huesos largos y diámetro máximo de las cabezas de húmeros y fémures.

\begin{tabular}{|c|c|c|c|c|c|}
\hline Elemento & $\begin{array}{c}\text { Longitud máx } \\
(\mathbf{m m})\end{array}$ & $\begin{array}{c}\text { Diám. máx de cabeza } \\
(\mathbf{m m})\end{array}$ & Elemento & $\begin{array}{c}\text { Longitud máx } \\
(\mathbf{m m})\end{array}$ & $\begin{array}{c}\text { Diám. máx de cabeza } \\
\text { (mm) }\end{array}$ \\
\hline Húmero Izq. & 286 & 42,58 & Húmero Der. & 300 & 44,99 \\
\hline Cúbito Izq. & 250,5 & - & Cúbito Der. & 258 & - \\
\hline Radio Izq. & 230 & - & Radio Der. & 238,5 & - \\
\hline Fémur Izq. & 435 & 45,43 & Fémur Der. & 432,5 & 45,89 \\
\hline Tibia Izq. & 365 & - & Tibia Der. & 367,5 & - \\
\hline Peroné Izq. & 355,5 & - & Peroné Der. & 363 & - \\
\hline
\end{tabular}


La longitud máxima del fémur permitió realizar una nueva estimación de estatura, en esta oportunidad, empleando una fórmula de regresión generada específicamente para poblaciones prehispánicas de Chubut y Santa Cruz por Béguelin (2005). Así de acuerdo con dicha fórmula la estatura del individuo de Cerro Yanquenao podría estimarse en $165 \mathrm{~cm}$ $\pm 2,72$. Esta estimación es similar a la realizada previamente $(166 \mathrm{~cm})$ a partir de la tabla publicada a finales del siglo XIX por Manouvrier que establecía la correlación entre la longitud de los huesos largos y la estatura en individuos de ascendencia europea. Se confirma también la observación hecha por las investigadoras sobre una diferencia apreciable en las dimensiones de los huesos largos de un lateral respecto del otro; el derecho, de mayores dimensiones que el izquierdo. Esta diferencia es más marcada en el húmero y en el peroné. No obstante, estudios recientes (Béguelin 2009) indican que los valores obtenidos entran dentro de los rangos de asimetría observados en otras muestras osteológicas de Chubut. Por último, se presentan también los datos de diámetro máximo de cabeza de fémur y húmero (Tabla 2) ya que contribuyen a generar una base de datos regional para esta medida que se presenta de utilidad para la generación de estándares locales para la estimación de sexo cuando están ausentes otros elementos diagnósticos (e.g. Bernal et al. 2004; García Guraieb et al. 2007).

\section{Análisis Paleopatológico}

Análisis paleopatológico bucal: En términos generales, ambos maxilares muestran tanto pérdidas postmortem como antemortem, éstos últimos en diferentes etapas de la vida del individuo a juzgar por los distintos estadios de remodelación del tejido óseo alveolar. En las piezas de ambas arcadas dentarias se observa un alto grado de desgaste que elimina completamente el esmalte en la mayoría de las piezas presentes exceptuando los segundos y terceros molares donde todavía se advierten bordes de esmalte que cubren total o parcialmente su perímetro. El maxilar superior muestra un proceso periodontal generalizado que produce la reabsorción total o parcial del tejido de sostén a lo largo de la arcada dentaria. Este proceso posiblemente se haya acoplado con la ocurrencia de abscesos locales causados por la exposición de la cámara pulpar en aquellas piezas permanentes de más temprana erupción (e.g. primeros molares, que son a su vez los que muestran secuelas de exfoliación más tempranas y un grado más avanzado de reabsorción del tejido alveolar). En esta arcada sólo se observan in situ el tercer molar derecho y el canino izquierdo. El maxilar inferior, presenta in situ la mayoría de las piezas dentarias. Se advierte la ausencia de ambos incisivos centrales, del incisivo lateral izquierdo y del primer y segundo molar derechos. A excepción del incisivo lateral inferior izquierdo, el grado de remodelación del tejido de sostén de las piezas faltantes indicaría que se trata de pérdidas antemortem. En ninguna de las piezas presentes en el maxilar superior o inferior se advirtió la presencia de caries. Exceptuando el proceso periodontal observado en el maxilar superior, los restantes patrones de salud bucal observados en este individuo son similares a los registrados en otras muestras de cazadores recolectores tardíos de Patagonia continental (García Guraieb 2004, García Guraieb y Maldonado 2007) y otras regiones áridas del país (Bernal et al. 2007)

Análisis paleopatológico óseo: Las lesiones óseas más conspicuas en el individuo de Cerro Yanquenao se observaron en el cráneo y en los huesos largos. En el cráneo, en el hueso frontal, a unos $30 \mathrm{~mm}$ de la glabela, se registró una imagen osteolítica de forma, borde y fondo irregulares de ca. $25 \mathrm{~mm}$ de diámetro (Fig.1). La lesión es somera, mostrando una profundidad máxima de 5 $\mathrm{mm}$ respecto de la tabla externa del cráneo. En el sector inferior de esta lesión, se advierte una zona

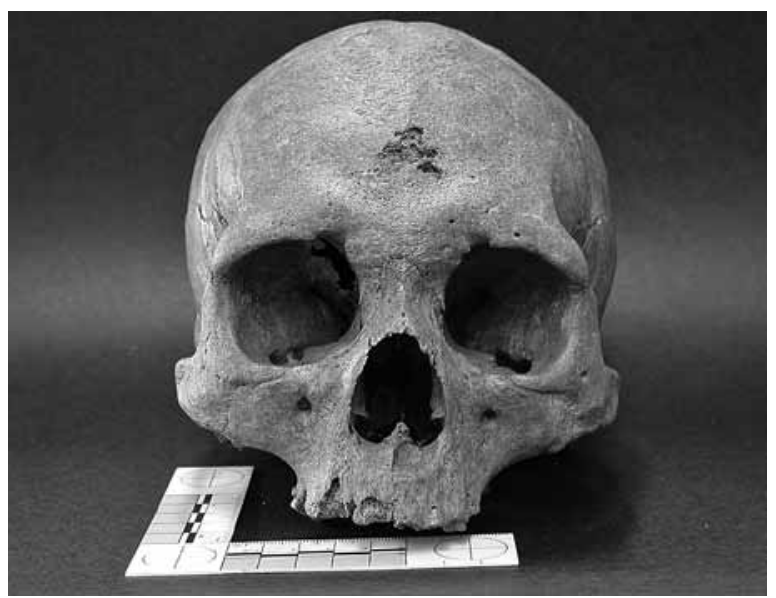

Fig. 1. Imagen osteolítica en el frontal y leve remodelación la región nasal. 


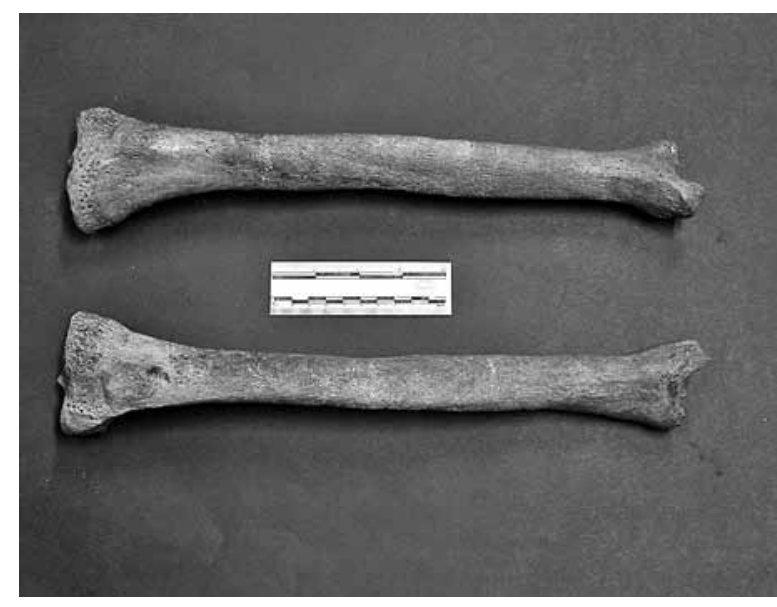

Fig. 2. Ambas tibias con periostitis/osteitis que afectan el contorno de las diáfisis.

de porosidad que se extiende hacia la glabela. La buena conservación del esqueleto en general y del cráneo en particular, contribuirían a descartar la idea previamente postulada de que se trate de un daño postmortem (Patti de Martínez Soler y Vayá 1986). La irregularidad de sus bordes y la falta de evidencias -a nivel macroscópico- de remodelación ósea, sugerirían que se trata de una lesión activa al momento de la muerte. Asimismo, en la zona nasal se observa una leve reabsorción y remodelación de los bordes ventrales de los nasales, la fosa nasal del maxilar superior y del tabique.

En el postcráneo, se observó una marcada reacción subperióstica/osteitis bilateral en tibias, fíbulas, cúbitos y radios. La reacción es simétrica al nivel de los miembros inferiores y afecta toda la diáfisis de las tibias (Fig. 2) y la diáfisis distal de los peronés. En el caso de los cúbitos y radios, las lesiones se manifiestan de forma bilateral pero asimétrica. Así, se observan lesiones en la diáfisis media y distal del cúbito y radio derechos, en la diáfisis media y proximal del cúbito izquierdo y en la diáfisis distal del radio izquierdo (Fig. 3). En ninguno de los elementos afectados, ya sean de los miembros inferiores o de los miembros superiores, se observan lesiones a nivel de las epífisis. En la mayoría de los casos, el nuevo hueso cortical es masivo y genera alteración del contorno del hueso, lo que le da al hueso un aspecto hinchado en las zonas afectadas. Asimismo, en la mayoría de los sectores, la lesión se encuentra remodelada o en proceso de remodelación por lo que la superficie se presenta finamente estriada. En

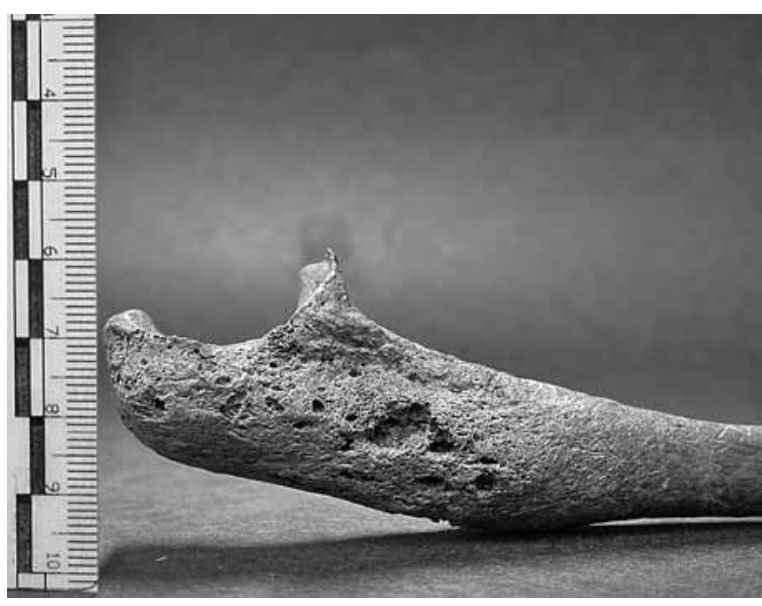

Fig. 3. Detalle de lesiones proliferativas y osteolítcas en cúbito izquierdo, sector póstero-lateral.

otros sectores, como el caso del cúbito izquierdo proximal póstero-lateral, las lesiones muestran un aspecto fibroso y focos líticos asociados.

Entre los diagnósticos diferenciales que podrían explicar la distribución y las características observadas en las lesiones de los huesos largos, se contemplaron: la osteoatropatía hipertrófica secundaria $^{3}$, la estasis venosa ${ }^{4}$, la osteomielitis inespecífica o alguna variedad de treponematosis. Esta última sería por el momento la que más se ajustaría al patrón de lesiones observado en los miembros inferiores y superiores del post-cráneo y permitiría explicar, además, la naturaleza de las leves lesiones observadas macroscópicamente en el cráneo (frontal, nasal y reabsorción periodontal). La presencia precolombina de distintas variedades de treponematosis ha sido identificada en numerosas muestras osteológicas en distintas regiones del continente americano desde hace varias décadas (Powell y Cook 2005, Standen y Arriaza 2000, entre otros). En Patagonia, su presencia también ha sido detectada osteológicamente, en años recientes, en colecciones correspondientes a grupos cazadores- recolectores del Holoceno tardío, tanto en Argentina (el individuo del sitio Carsa, en la

3 La osteoartropatía hipertrófica secundaria puede ser causada por tumores o patologías pulmonares que pueden ocasionar periostitis difusa en tibias, peronés, radios y cúbitos, entre otros síntomas clínicos (Resnick 2001: 1220).

4 En la medida en que la estasis venosa (várices) se acompaña de hiperemia pasiva (i.e. una disminución del flujo sanguíneo por un bloqueo u obstrucción del mismo) también puede producir depositación de hueso nuevo en los miembros inferiores (Knüsel y Ogden 2008). 
costa septentrional de Santa Cruz; Castro et al. 2008) como en Chile (muestras correspondientes grupos denominados como "Chonos", de las islas Guaitecas en el sur del archipiélago de Chiloe; Aspillaga et al. 2006, Rodríguez et al. 2007). Sin embargo, estos casos o bien corresponden a momentos posteriores al contacto europeo o bien no cuentan aún con fechados radiocarbónicos directos. En este contexto, cobra especial importancia, la profundización en el diagnóstico diferencial del caso de Cerro Yanquenao, mediante técnicas paleopatológicas alternativas, como la radiografía convencional y la tomografía computada, que contribuyan a dilucidar la naturaleza de las lesiones craneales y post-craneales y evaluar la pertinencia del caso en la discusión sobre la epidemiología de la treponematosis en Patagonia precolombina 5 .

Para concluir, cabe mencionar otras manifestaciones paleopatológicas menos conspicuas, presentes en este caso. En todos los cuerpos vertebrales presentes -incluyendo el primer fragmento del sacro-se observaron osteofitos ${ }^{6}$ marginales horizontales y verticales de diverso grado de desarrollo, con predominio hacia el lateral derecho. Los mismos serían consistentes con un cuadro de osteocondrosis vertebral ${ }^{7}$. Asimismo, se hallaron osteofitos marginales en las superficies articulares de los procesos transversos de las vértebras lumbares y algunas de las toráxicas presentes, que sugerirían la incipiente osteoartrosis de estas articulaciones. Estas artropatías, son desarrollos comunes en individuos mayores de

5 Estos estudios más específicos, que requieren de la colaboración con otros especialistas (por ejemplo, especialistas en diagnósticos por imágenes), se encuentran aún en curso y sus resultados serán presentados en otra oportunidad en el marco de una discusión estrictamente paleopatológica.

6 Se denomina osteofitos a la excrecencias óseas en los huesos del cuerpo. En las articulaciones sinoviales, los osteofitos suelen originarse en sus márgenes, en el punto en el que la membrana sinovial es continua con el cartílago articular. En los cuerpos vertebrales los osteofitos surgen del punto de inserción de las fibras del anillo fibroso vertebral. Los osteofitos tienden a desarrollarse horizontalmente, pero pueden volverse verticales si crecen lo suficiente. La osteofitosis es frecuente en distintas enfermedades reumáticas y es común encontrarla asociada al envejecimiento (Rogers y Waldron 1995).

7 Las lesiones del cartílago intervertebral, llamadas también osteocondrosis, consisten en la degeneración aislada de un disco intervertebral. Es una afección frecuente, pero raras veces adopta formas graves.
40 años. En este sentido, la estimación de una edad de entre ca. 40-50 años para el individuo es más congruente con la presencia de esta condición que la previamente reportada (ca. 25-30 años; Patti de Martínez Soler y Vayá 1986: 42).

También se registró un osteoma en forma de botón en el parietal derecho (norma posterior). Estos pequeños tumores benignos, son más frecuentes en los individuos masculinos que en los femeninos, suelen aparecer con mayor frecuencia entre la cuarta y quinta década de vida del individuo (Aufderheide y Rodríguez-Martín 1998) y no tienen significado patológico. Por último, no se hallaron evidencias de criba orbitaria, hiperostosis porótica, hipoplasias lineales de esmalte dental, ni traumas.

\section{CONSIDERACIONES FINALES}

Desde el momento del hallazgo del entierro de Cerro Yanquenao, hace casi treinta años, se han realizado, en distintos sectores de Patagonia continental austral, numerosas investigaciones sistemáticas que han generado un importante volumen de información bioarqueológica y han estado guiadas por nuevos problemas de investigación y modelos explicativos. En el fortalecimiento y construcción de estos modelos cobran interés tanto los hallazgos arqueológicos concentrados como los casos aislados. Hasta el presente, el tipo de información disponible para el esqueleto de Cerro Yanquenao había limitado la posibilidad de incorporar el caso en las discusiones bioarqueológicas regionales de los últimos años. La obtención de una cronología radiocarbónica para el entierro, los nuevos datos isotópicos sobre dieta y la reevaluación del perfil biológico del individuo permiten ahora incluir este hallazgo dentro del conjunto de información disponible para la región. En este sentido, se puede observar que el entierro de Cerro Yanquenao presenta características similares -en cuanto a la modalidad de entierro en chenques, los valores isotópicos, el tipo de deformación craneana artificial- a otros hallazgos de la región, asignables a la misma cronología. Asimismo, el nuevo análisis paleopatológico permitió observar patrones comunes con otras muestras osteológicas de cronología y contexto homologables (por ejemplo en los patrones de salud bucal) e identificar lesiones óseas que no habían sido detectadas en el análisis previo del esqueleto. Las mismas aportan nuevos datos a las 
problemáticas sobre los patrones de salud general de los grupos cazadores recolectores patagónicos en tiempos prehispánicos $y$, de contrastarse el diagnóstico de treponematosis mediante la profundización de los estudios, será posible contar con información fundamental para la comprensión de la distribución geográfica y temporal de este grupo de enfermedades en Sudamérica. En suma, los nuevos análisis realizados sobre el individuo recuperado en Cerro Yanquenao, ha permitido actualizar la información disponible e incluir este importante hallazgo, en términos de su completitud y contexto conocido, dentro de las discusiones vigentes sobre la bioarqueología de los grupos cazadores-recolectores patagónicos del Holoceno tardío.

\section{AGRADECIMIENTOS}

Al Dr. T. Jull del Laboratorio de Arizona (USA) por los análisis efectuados. Los mismos fueron financiados por el Proyecto PIP-CONICET 02466/ 2004. A la Dra. M. Béguelin por permitirnos emplear datos provenientes de su tesis Doctoral. A M. Plischuk y B. Desántolo por facilitarnos el trabajo en prensa del sitio Carsa. A los Dres. J. Pereyra, S. Mendonça de Souza y F. J. Aguerre por sus aportes en la discusión paleopatológica del caso.

\section{BIBLIOGRAFÍA CITADA}

ACSÁDI, G. y J. NEMESKÉRI. 1970. History of Human Life Span and Mortality. Budapest, Academia Kiado.

ASPILlAGA, F.E, M. CASTRO D., M. RODRÍGUEZ, y C. OCAMPO E. 2006. Paleopatología y estilo de vida: el ejemplo de los Chonos. Magallania 34(1): 77-85. Punta Arenas. Chile.

AUFDERHEIDE, A. y C. RODRÍGUEZ-MARTÍN. 1998. The Cambridge Encyclopedia of Human Paleopathology. Cambridge University Press. Cambridge.

BÉGUELIN, M. 2005. Estimación de la estatura en muestras del Holoceno Tardío Del N.O. de Santa Cruz: problemas metodológicos. Revista Argentina de Antropología Biológica 7 (1): 108.

BÉGUELIN, M. 2009. Variación Geográfica en la Morfología del Esqueleto Postcraneal de las Poblaciones Humanas de Pampa y Patagonia durante el Holoceno Tardío: Una Aproximación Morfométrica. Tesis de Doctorado en Ciencias Naturales. Facultad de Ciencias Naturales y Museo. Universidad Nacional de La Plata.
BÉGUELIN, M. y P. N. GONZALEZ. 2008. Estimación del sexo en poblaciones del sur de Sudamérica mediante funciones discriminantes para el fémur. Revista Argentina de Antropología Biológica 10(2): 55-70.

BERNAL, V. y A. M. AGUERRE. 2008. Investigaciones Bioarqueológicas en Río Mayo (SO de Chubut). En: Huellas del hombre en el sudoeste de Chubut, C. Pérez de Micou, L.S. Burry y M. Trivi (editores). En prensa.

BERNAL, V., S. GARCÍA GURAIEB y P. N. GONZÁLEZ. 2004. Perfiles de mortalidad de las muestras de restos humanos procedentes del área del Lago Salitroso (Provincia de Santa Cruz). En Contra viento y Marea. Arqueología de Patagonia. M.T. Civalero, P.M. Fernandez y A.G. Guráieb (compiladores); pp 361-373. Instituto Nacional de Antropología y Pensamiento Latinoamericano. Sociedad Argentina de Antropología, Buenos Aires.

BERNAL V.; NOVELLINO P.; GONZALEZ P.; PEREZ S.I. 2007. Role of wild plant foods among Late Holocene huntergatherers from Central and North Patagonia (South America): an approach from dental evidence. American Journal of Physical Anthropology 133:1047-1059.

BÓRMIDA, M. 1953-1954. Los antiguos patagones. Estudio de craneología. RUNA. Archivo para las Ciencias del Hombre. 6:5-96. Buenos Aires.

BUIKSTRA, J. y D. UBELAKER. 1994. Standards for data collection from human skeletal remains. Archaeological Survey Research Series 44. Arkansas.

CLARKE, N. y R. HIRSCH. 1991. Physiological, pulpal and periodontal factors influencing alveolar bone. En: Advances in Dental Anthropology. A. Kelley y C. S. Larsen (editores) pp. 241-66. Willey-Liss. New York.

CASTRO, A., SALCEDA, S. PLISCHUK, M. y B. DESÁNTOLO. 2008. Bioarqueología de rescate: Sitio Carsa (Costa Norte de Santa Cruz, Argentina). "Arqueología de Patagonia: una mirada desde el último confín”, M. Salemme, F. Santiago, M. Alvarez, E. Piana, M. Vázquez y E. Mansur (editores). Editorial Utopías (Ushuaia). En prensa.

FERNANDEZ J. y H. PANARELLO. 1994. Estimaciones paleodietéticas y ambientales, esqueletos 1 y 2 Puesto $\mathrm{El}$ Rodeo. Análisis de las relaciones isotópicas $13 \mathrm{C} / 12 \mathrm{C}$ en el colágeno. En: Contribución a la arqueología del Río Pinturas, Provincia de Santa Cruz. C.J. Gradin y A.M. Aguerre (editores); pp. 300- 310. Ediciones Búsqueda de Ayllu, Concepción del Uruguay, Argentina.

GARCÍA GURAIEB S. 2004. Salud y enfermedad en cazadoresrecolectores del Holoceno tardío en la cuenca del lago Salitroso (Santa Cruz). Tesis de Licenciatura en Ciencias Antropológicas (orientación Arqueología). Facultad de Filosofía y Letras. Universidad de Buenos Aires. Ms. 
GARCÍA GURAIEB S. 2006. Salud y enfermedad en cazadoresrecolectores del Holoceno tardío en la cuenca del Lago Salitroso (Santa Cruz). Intersecciones en Arqueología 7:37-48. UNC-Olavarría.

GARCÍA GURAIEB, S y M. B. MALDONADO. 2007. Estudio de adecuación fisiológica y salud bucal de restos óseos de cazadores-recolectores del Holoceno tardío del lago Salitroso (Noroeste de Santa Cruz). Revista Argentina de Antropología Biológica 9(1): 98.

GARCÍA GURAIEB, S., P. GONZALEZ y V. BERNAL. 2007.

Estructura de sexo y edad de la muestra de restos humanos del holoceno tardío del lago salitroso (Santa Cruz, Argentina). En: Arqueología de Fuego-Patagonia. Levantando piedras, desenterrando huesos... y develando arcanos. F. Morello, M. Martinic, A. Prieto y G. Bahamonde (editores); pp. 367-374. Ediciones CEQUA: Punta Arenas.

GOMEZ OTERO, J. y S. DAHINTEN. 1997-1998. Costumbres funerarias y esqueletos humanos: variabilidad y poblamiento en la costa Nordeste de la Provincia del Chubut (Patagonia Argentina). Relaciones 22-23:101124. Buenos Aires.

GOÑI, R. A, G. BARRIENTOS y G. CASSIODORO. 2000-2002. Condiciones previas a la extinción de las poblaciones humanas del sur de Patagonia: una discusión a partir del análisis del registro arqueológico de la cuenca del lago Salitroso. Cuadernos del Instituto Nacional de Antropología y Pensamiento Latinoamericano 19:249-266.

GOÑI, R. A. y G. BARRIENTOS. 2004. Poblamiento Tardío y movilidad en la cuenca del lago Salitroso. En: Contra Viento y Marea. M. T. Civalero, P. Fernández y A. G. Guraieb (compiladores); pp.313-324. Buenos Aires.

GRADIN, C. J. 1986. Los grabados rupestres del cerro Yanquenao (Chubut). Publicación del Gobierno de la Provincia del Chubut, Rawson.

GRADIN, C. J. y A.M. AGUERRE. 1994. Excavación del enterratorio del Puesto "El Rodeo". En: Contribución a la arqueología del Río Pinturas, Provincia de Santa Cruz. C.J. Gradin y A. M. Aguerre (editores); pp. 259272.Ediciones Búsqueda de Ayllu, Concepción del Uruguay, Argentina.

HILLSON, S. 2000. Dental Pathology. En: Biological Anthropology of the Human Skeleton. M. A. Katzemberg y S.R. Saunders (editores); pp. 249-286. Willey-Liss.

IMBELLONI, J. 1923. Habitantes neolíticos del Lago Buenos Aires. Documentos para la antropología física de la Patagonia Austral. Revista del Museo de La Plata 27: 85-160.
IMBELLONI, J. 1924-25. Deformaciones intencionales del cráneo en Sudamérica. Revista del Museo de La Plata 28: 329-407.

KNÜSEL C. J. y A. R. OGDEN. 2008. Paleopathology. En: Encyclopedia of Archaeology, vol. 3. D.M. Pearsall (editora); pp. 1795-1809. Academic Press, New York.

LOVEJOY, O., R. MEINDL, T. PRYZBECK, y R. MENSFORTH. 1985. Chronological metamorphosis of the auricular surface of the ilium: a new method for the determination of adult skeletal age at death. American Journal of Physical Anthropology 68:15-28.

LOVELL, N. 2000. Paleopathological description and diagnosis. En: Biological Anthropology of the human skeleton.

M. Katzenberg y S. Saunders (editors); pp. 217-248. Willey-Liss Inc.

McCORMAC F.G., A.G. HOGG, P.G. BLACKWELL, C.E. BUCK, T.F.G. HIGHAM y PJ REIMER. 2004. SHCal04 Southern Hemisphere Calibration 0-11.0 cal kyr BP. Radiocarbon 46: 1087-1092

MEINDL, R. y O. LOVEJOY. 1985. Ectocranial suture closure: a revised method for the determination of skeletal age at death based on the lateral-anterior sutures. American Journal of Physical Anthropology 68: 57-66

MEINDL, R. y O. LOVEJOY. 1989. Age changes in the pelvis: implications for paleodemography. En: Age markers in the human skeleton. M.Y. Iscan (editor); pp. 137-168. Springfield IL: Charles C Thomas, 1989.

MIRANDA, S. 1986. Características morfológico-dentarias del cráneo del Cerro Yanquenao- Provincia del Chubut. En Grabados rupestres del Cerro Yanquenao en la Provincia del Chubut. C. J. Gradin (editor); pp. 61-62. Publicación del Gobierno de la Provincia del Chubut, Rawson

PATTI DE MARTINEZ SOLER J. y C. VAYÁ. 1986. Estudios del esqueleto humano del cerro Yanquenao, Provincia del Chubut. En: Grabados rupestres del Cerro Yanquenao en la Provincia del Chubut. C. J. Gradin; pp 37-59. Publicación del Gobierno de la Provincia del Chubut, Rawson.

PEREZ, S. I. 2007. Artificial cranial deformation in South America: a geometric morphometrics approximation. Journal of Archaeological Science 34: 1649-1658.

PHENICE, T. 1969. A newly development visual method of sexing the os pubis. American Journal of Physical Anthropology. 30: 297-301.

POWELL, M. L. y D. C. COOK. 2005. The Myth of syphilis. The Natural history of treponematosis in NorthAmerica. University Press of Florida. 
RESNICK, D. 2001. Enostosis, hiperostosis y periostitis. En. Huesos y articulaciones en imagen. D. Resnick (editor) $2^{a}$ edición; pp. 1211-1231. Marbán. Madrid.

RODRÍGUEZ, M., E. ASPILLAGA y B. ARENSBURG. 2007. El estudio bioantropológico de las colecciones esqueletales del archipiélago de Chiloé: perspectivas y limitaciones. En: Arqueología de Fuego-Patagonia. Levantando piedras, desenterrando huesos...y develando arcanos. F. Morillo, M. Martinic, A.Prieto y G. Bahamonde; pp. 269-278. Ediciones CEQUEA. Punta Arenas, Chile.

ROGERS, J. y T. WALDRON. 1995. A field guide to joint disease in archaeology. John Wiley \& Sons.

SCOTT, E. C. 1979. Dental wear scoring technique. American Journal of Physical Anthropology 51: 213-218.

SMITH, B. H. 1984. Patterns of molar wear in hunter-gatherers and agriculturalists. American Journal of Physical Anthropology. 63:39-56.
STANDEN, V. G. y B. T. ARRIAZA. 2000. La treponematosis (Yaws) en las poblaciones prehispánicas del desierto de Atacama. Chungara 32(2): 185-192.

STUIVER, M. y P. J. REIMER. 1993. Extended 14C data base and revised CALIB $3.014 \mathrm{C}$ age calibration program. Radiocarbon 35:215-230.

TESSONE, A., A. ZANGRANDO, G. BARRIENTOS, S. VALENCIO, H. PANARELLO y R. GOÑI. 2005. Isótopos estables del carbono en Patagonia Meridional: datos de la Cuenca del Lago Salitroso (Provincia de Santa Cruz, República Argentina). Magallania 33: 21-28.

UBELAKER, D. H. 1989 Human Skeletal Remains: Excavation, Analysis, Interpretation. Taraxacum, Washington. 
